\title{
ON A CHARACTERISATION OF DIFFERENTIABILITY OF THE NORM OF A NORMED LINEAR SPACE
}

\author{
J. R. GILES \\ (Received 5 March 1969) \\ Communicated by J. B. Miller
}

The purpose of this paper is to show that the various differentiability conditions for the norm of a normed linear space can be characterised by continuity conditions for a certain mapping from the space into its dual. Differentiability properties of the norm are often more easily handled using this characterisation and to demonstrate this we give somewhat more direct proofs of the reflexivity of a Banach space whose dual norm is strongly differentiable, and the duality of uniform rotundity and uniform strong differentiability of the norm for a Banach space.

The author wishes to express his thanks to Professor G. Szekeres under whose supervision part of this work was done towards a doctoral thesis submitted to the University of Newcastle, N.S.W.

\section{The characterisation of differentiability of the norm}

Notation. We denote by

$X \quad$ a normed linear space over the real or complex numbers;

$X^{*}$ the dual space of $X$, and $X^{* *}$ the dual of $X^{*}$;

$\hat{X} \quad$ the natural embedding of $X$ in $X^{* *}$;

$S \quad$ the unit sphere of $X$; i.e. $S \equiv\{x \in X:\|x\|=1\}$;

$B \quad$ the unit ball of $X$; i.e. $B \equiv\{x \in X:\|x\| \leqq 1\}$;

$S^{*}, \quad B^{*}, S^{* *}, B^{* *}, \widehat{S}, \widehat{B}$ the unit sphere and unit ball of $X^{*}, X^{* *}, \hat{X}$ respectively; $D(x)$ the set of $f \in S^{*}$ which attain their norm at $x \in S$, i.e. given $x \in S$, $D(x) \equiv\left\{f \in S^{*}: f(x)=\|f\|\right\}$; (by the Hahn-Banach theorem, $D(x)$ is non-empty for each $x \in S$ ).

Definitions. We say that

$X$ is smooth at $x \in S$ if $D(x)$ contains only one point;

a mapping $x \rightarrow f_{x}$ of $X$ into $X^{*}$ is a support mapping if, for each $x \in S$ 
and real $\lambda \geqq 0, f_{x} \in D(x)$ and $f_{\lambda x}=\lambda f_{x}$ (in general there are many such mappings possible on $X$ but when $X$ is smooth there is only one);

the norm of $X$ is

(i) weakly (Gâteaux) differentiable at $x \in S$ if for real $\lambda$,

$$
\lim _{\lambda \rightarrow 0} \frac{\|x+\lambda y\|-\|x\|}{\lambda}
$$

exists for all $y \in S$,

(ii) strongly (Fréchet) differentiable at $x \in S$ if convergence to the limit in (i) is uniform for all $y \in S$,

(iii) uniformly strongly (uniformly Fréchet) differentiable if convergence to the limit in (i) is uniform for all $(x, y) \in S \times S$;

$X$ is uniformly smooth if, given $\varepsilon>0$ there exists a $\delta>0$ such that for every $x \in S$

$$
\|x+y\|+\|x-y\|<2+\varepsilon\|y\| \text { when }\|y\|<\delta ;
$$

$X$ is uniformly rotund if, given $\varepsilon>0$ there exists a $\delta>0$ such that, for $x, y \in S,\|x-y\|<\varepsilon$ when $\|x+y\|>2-\delta$.

Our aim is to establish the following result:

THEOREM 1. The norm of $X$ is

(i) weakly differentiable at $x \in S$ if and only if, when $X$ has the norm topology and $X^{*}$ the weak * topology, there exists a support mapping $x \rightarrow f_{x}$ which is continuous on $S$ at $x$,

(ii) strongly differentiable at $x \in S$ if and only if, when $X$ and $X^{*}$ have the norm topologies, there exists a support mapping $x \rightarrow f_{x}$ which is continuous on $S$ at $x$,

(iii) uniformly strongly differentiable if and only if, when $X$ and $X^{*}$ have the norm topologies, there exists a support mapping $x \rightarrow f_{x}$ which is uniformly continuous on $S$.

V. L. Smulian has given similar equivalent conditions [6, p. 91], [7, p. 645] for differentiability of the norm. However several recent developments have suggested that some of Šmulian's work can be extended and proved by simpler methods. D. F. Cudia [3, pp. 297-307] has lately shown that differentiability properties of the norm are closely related to continuity properties of a certain set-valued mapping which he calls a spherical image map. When the space is smooth, the support mapping corresponds to the spherical image map. Part (iii) of the theorem has been given by Cudia [3, p. 304] but parts (i) and (ii) are a local form of his global results [3, p. 302 and p. 304], and as such are an improvement of his work. But the techniques associated with the support mapping certainly have the advantage in providing a simple and direct proof. 
The proof of Theorem 1 will be apparent from the following sequence of lemmas.

We begin by showing that the differentiability properties of the norm are implied by corresponding continuity properties for the support mapping.

LEMMA 1. The norm of $X$ is

(i) weakly differentiable at $x \in S$ if there exists a support mapping $x \rightarrow f_{x}$ on $X$ such that, for real $\lambda$,

for all $y \in S$.

$$
\lim _{\lambda \rightarrow 0} \operatorname{Re}\left\{\frac{f_{x+\lambda y}(y)}{\|x+\lambda y\|}-\frac{f_{x}(y)}{\|x\|}\right\}=0
$$

(ii) strongly differentiable at $x \in S$ if convergence to the limit in (i) is uniform for all $y \in S$;

(iii) uniformly strongly differentiable if convergence to the limit in (i) is uniform for all $(x, y) \in S \times S$.

Proof. Given any support mapping $x \rightarrow f_{x}$ on $X$, then for $x, y \in S$ and real $\lambda>0$,

$$
\begin{aligned}
\frac{\|x+\lambda y\|-\|x\|}{\lambda} & \geqq \frac{\left|f_{x}(x+\lambda y)\right|-\|x\|^{2}}{\lambda\|x\|} \\
& \geqq \frac{\operatorname{Re}\left\{f_{x}(x+\lambda y)\right\}-\|x\|^{2}}{\lambda\|x\|} \\
& =\frac{\operatorname{Re}\left\{f_{x}(y)\right\}}{\|x\|}
\end{aligned}
$$

But also

$$
\begin{aligned}
\frac{\|x+\lambda y\|-\|x\|}{\lambda} & \leqq \frac{\|x+\lambda y\|^{2}-\left|f_{x+\lambda y}(x)\right|}{\lambda\|x+\lambda y\|} \\
& \leqq \frac{\operatorname{Re}\left\{f_{x+\lambda y}(x)+\lambda f_{x+\lambda y}(y)\right\}-\left|f_{x+\lambda y}(x)\right|}{\lambda\|x+\lambda y\|} \\
& \leqq \frac{\operatorname{Re}\left\{f_{x+\lambda y}(y)\right\}}{\|x+\lambda y\|} .
\end{aligned}
$$

For $\lambda<0$ these inequalities are reversed. It follows that if a support mapping satisfies continuity conditions (i), (ii) or (iii) then the norm satisfies differentiability conditions (i), (ii) or (iii) respectively, and the Gâteaux differential of the norm at $x$ in the direction $y$ is $\operatorname{Re}\left\{f_{x}(y)\right\} /\|x\|$.

It is evident that this lemma establishes the sufficiency conditions for (i), (ii) and (iii) of Theorem 1.

We will need the following well known result. 
LEMMA 2. If the norm of $X$ is weakly differentiable at $x \in S$ then $X$ smooth at $x$.

Proof. From inequality (1) we deduce that, for any $y \in S$ and all $f \in D(x)$,

$$
\frac{\|x+\lambda y\|-\|x\|}{\lambda} \leqq \operatorname{Re}\{f(y)\} \quad \text { for } \lambda<0
$$

and

$$
\operatorname{Re}\{f(y)\} \leqq \frac{\|x+\lambda y\|-\|x\|}{\lambda} \quad \text { for } \lambda>0 .
$$

But $\lim _{\lambda \rightarrow 0}\{\|x+\lambda y\|-\|x\|\} / \lambda$ exists, and so $\operatorname{Re}\{f(y)\}$ is constant for all $f \in D(x)$. As every $f \in X^{*}$ can be defined in terms of $\operatorname{Re}\{f\}$, it follows that $D(x)$ is singleton, i.e. $X$ is smooth at $x$.

The following is an adaptation of an argument of F. F. Bonsall, B. E. Cain and H. Schneider [2, p. 88].

LEMma 3. If $X$ is smooth at $x \in S$ then every support mapping $x \rightarrow f_{x}$ is continuous on $S$ at $x$ when $X$ has the norm topology and $X^{*}$ has the weak ${ }^{*}$ topology.

Proof. Suppose that there exists a support mapping $x \rightarrow f_{x}$ which is not continuous on $S$ at $x$. Then there exists a weak * neighbourhood $U$ of $f_{x}$ such that, for every positive integer $n$, there exists a $y_{n} \in S$ satisfying $f_{y_{n}} \notin U$ when $\left\|y_{n}-x\right\|<1 / n$. By the Banach-Alaoglu theorem $B^{*}$ is weak * compact. Since $f_{v_{v}} \in S^{*}$ for every $n$, there exists for $\left\{f_{y_{n}}\right\}$ a weak $*$ cluster point $f \in B^{*}$. But

$$
\begin{aligned}
|f(x)-1| & \leqq\left|f(x)-f_{y_{n}}(x)\right|+\left|f_{y_{n}}(x)-f_{y_{n}}\left(y_{n}\right)\right| \\
& \leqq\left|f(x)-f_{y_{n}}(x)\right|+|| x-y_{n}|| .
\end{aligned}
$$

Since $f$ is a weak * cluster point of $\left\{f_{y_{n}}\right\}$ and $\left\|x-y_{n}\right\|<1 / n$, then $|f(x)-1|$ can be made arbitrarily small for proper choice of $n$. Therefore $f(x)=1$ and $f \in D(x)$. Since $X$ is smooth at $x, f=f_{x}$. Therefore $f_{x}$ is a weak * cluster point for $\left\{f_{y_{n}}\right\}$. But $U$ is a weak * neighbourhood of $f_{x}$ so $f_{y_{n}} \in U$ for some $n$. But this is a contradiction. Therefore every support mapping $x \rightarrow f_{x}$ is continuous on $S$ at $x$.

The necessity condition for (i) of Theorem 1 . follows immediately from Lemmas 2 and 3.

We now explore an implication of strong differentiability of the norm. The result was given by Smulian [7, p. 645] but we follow the simpler proof indicated by R. R. Phelps [5, p. 981].

LEMMA 4. If the norm of $X$ is strongly differentiable at $x \in S$, then all sequences $\left\{f_{n}\right\} \in S^{*}$ such that $\lim _{n \rightarrow \infty} f_{n}(x)=\|x\|$, are norm convergent to $f_{x}$ where $D(x)=\left\{f_{x}\right\}$.

Proof. It is obvious that the norm is strongly differentiable at $x \in S$ if and only if 


$$
\lim _{y \rightarrow 0} \frac{\|x+y\|-\|x\|-\operatorname{Re}\left\{f_{x}(y)\right\}}{\|y\|}=0, \quad \text { for all } y \in X .
$$

Suppose that there exists such a sequence $\left\{f_{n}\right\}$ which is not norm convergent to $f_{x}$. Then for some subsequence $\left\{f_{n_{k}}\right\}$ we can choose $r>0$ and a sequence $\left\{y_{k}\right\} \in S$ such that $\operatorname{Re}\left\{\left(f_{n_{k}}-f_{x}\right)\left(y_{k}\right)\right\}>2 r$. For every sequence $\left\{x_{k}\right\} \in S$,

$$
\begin{aligned}
\left\|x+x_{k}\right\|-\|x\|-\operatorname{Re}\left\{f_{x}\left(x_{k}\right)\right\} & \geqq\left|f_{n_{k}}\left(x+x_{k}\right)\right|-\operatorname{Re}\left\{f_{x}\left(x+x_{k}\right)\right\} \\
& \geqq \operatorname{Re}\left\{\left(f_{n_{k}}-f_{x}\right)\left(x+x_{k}\right)\right\} .
\end{aligned}
$$

Choose $x_{k}=(1 / r) \operatorname{Re}\left\{\|x\|-f_{n_{k}}(x)\right\} y_{k}$, then

Therefore

$$
\operatorname{Re}\left\{\left(f_{n_{k}}-f_{x}\right)\left(x+x_{k}\right)\right\} \geqq \operatorname{Re}\left\{\left(f_{x}-f_{n_{k}}\right)(x)\right\} .
$$

$$
\frac{\left\|x+x_{k}\right\|-\|x\|-\operatorname{Re}\left\{f_{x}\left(x_{k}\right)\right\}}{\left\|x_{k}\right\|} \geqq r
$$

for all such $x_{k}$ although $x_{k} \rightarrow 0$. But this contradicts the fact that the norm is strongly differentiable at $x$. Therefore $\left\{f_{n}\right\}$ is norm convergent to $f_{x}$.

If the norm of $X$ is strongly differentiable at $x \in S$ then it is weakly differentiable there and it follows from Theorem 1 (i) that there exists a support mapping $x \rightarrow f_{x}$ on $X$ which has the property that the sequence $\left\{f_{x_{n}}\right\}$ is weak * convergent to $f_{x}$ for all sequences $\left\{x_{n}\right\} \in S$ which are norm convergent to $x$. Therefore

$$
\lim _{n \rightarrow \infty} f_{x_{n}}(x)=\|x\|
$$

for all such sequences $\left\{x_{n}\right\}$ and by Lemma 4 . it follows that $\left\{f_{x_{n}}\right\}$ is norm convergent to $f_{x}$. That is, when $X$ and $X^{*}$ have the norm topologies then the support mapping $x \rightarrow f_{x}$ is continuous on $S$ at $x$. So then the necessity condition for (ii) of Theorem 1. is implied by Lemma 4 .

With some economy of argument the following lemma gives a considerable amount of information. The proof that property (iii) implies property (iv) is given by H. Nakano [4, p. 202] and is included for completeness sake and because it is so brief.

LEMMA 5. For $X$, the following conditions are equivalent:

(i) there exists a support mapping $x \rightarrow f_{x}$ which is uniformly continuous on $S$ when $X$ and $X^{*}$ have the norm topologies,

(ii) the norm of $X$ is uniformly strongly differentiable,

(iii) $X$ is uniformly smooth,

(iv) $X^{*}$ is uniformly rotund.

ProOF.

(i) $\Rightarrow$ (ii). We have mentioned that this result is a consequence of Lemma 1 (iii). 
(ii) $\Rightarrow$ (iii). Since the norm of $X$ is uniformly strongly differentiable, it follows that, given $\varepsilon>0$ there exists a $\delta>0$ such that

$$
\begin{aligned}
& \frac{\left|\|x+y\|-\|x\|-\operatorname{Re}\left\{f_{x}(y)\right\}\right|}{\|y\|}<\frac{\varepsilon}{2} \quad \text { and } \\
& \frac{\left|\|x-y\|-\|x\|+\operatorname{Re}\left\{f_{x}(y)\right\}\right|}{\|y\|}<\frac{\varepsilon}{2}
\end{aligned}
$$

for every $x \in S$ and $\|y\|<\delta$. Therefore

$$
\begin{aligned}
& \frac{\|x+y\|+\|x-y\|-2}{\|y\|}<\varepsilon, \quad \text { or } \\
& \|x+y\|+\|x-y\|<2+\varepsilon\|y\|
\end{aligned}
$$

for every $x \in S$ and $\|y\|<\delta$; i.e., $X$ is uniformly smooth.

(iii) $\Rightarrow$ (iv). Since $X$ is uniformly smooth, given $\varepsilon>0$ there exists a $\delta>0$ such that

$$
\|x+y\|+\|x-y\|<2+\frac{1}{4} \varepsilon\|y\|
$$

for $x \in S$ and $\|y\|<\delta$. Choose $f, g \in S^{*}$ such that $\|f-g\| \geqq \varepsilon$. From the definition of the norm of $X^{*}$, there exists a $y \in X$ such that $\|y\|=\delta / 2$ and

Then we have

$$
(f-g)(y)>\frac{1}{4} \varepsilon \delta \text {. }
$$

$$
\begin{aligned}
\|f+g\| & =\sup \{(f+g)(x): x \in S\} \\
& =\sup \{f(x+y)+g(x-y)-(f-g)(y): x \in S\} \\
& <\sup \left\{\|x+y\|+\|x-y\|-\frac{1}{4} \varepsilon \delta: x \in S\right\} \\
& <2+\frac{1}{4} \varepsilon\|y\|-\frac{1}{4} \varepsilon \delta, \\
& <2-\frac{1}{8} \varepsilon \delta .
\end{aligned}
$$

Therefore $X^{*}$ is uniformly rotund.

(iv) $\Rightarrow$ (i). For every support mapping $x \rightarrow f_{x}$ on $X$, the following primitive parallelogram law holds:

$$
2\left(\|x\|^{2}+\|y\|^{2}\right)=\left(f_{x}+f_{y}\right)(x+y)+\left(f_{x}-f_{y}\right)(x-y) .
$$

This implies that for $x, y \in S$

$$
4 \leqq\left\|f_{x}+f_{y}\right\|\|x+y\|+\left\|f_{x}-f_{y}\right\|\|x-y\| .
$$

But when $X^{*}$ is uniformly rotund then, given $\varepsilon>0$ there exists a $\delta>0$ such that, for $f_{x}, f_{y} \in S^{*}$,

$$
\left\|f_{x}-f_{y}\right\|<\varepsilon \text { when }\left\|f_{x}+f_{y}\right\|>2-\delta
$$


Therefore, when $\|x-y\|<\delta$,

$$
\begin{aligned}
2 & \leqq\left\|f_{x}+f_{y}\right\|+\|x-y\| \\
& <\left\|f_{x}+f_{y}\right\|+\delta
\end{aligned}
$$

i.e. $\left\|f_{x}+f_{y}\right\|>2-\delta$, and we conclude that $\left\|f_{x}-f_{y}\right\|<\varepsilon$. The support mapping $x \rightarrow f_{x}$ is uniformly continuous on $S$.

Lemma 5 includes the specific statement of (iii) of Theorem 1.

\section{Applications of the characterisation}

The characterisation of differentiability of the norm given in Theorem 1 is particularly valuable when we use it in conjunction with the subreflexivity property of Banach spaces. Using this technique we establish some important known results in an elementary way.

Notation. We denote by $D(S)$ the set of $f \in S^{*}$ which attain their norm on $S$, i.e.

$$
D(S) \equiv U\{D(x): x \in S\} .
$$

Definition. We say that $X$ is subreflexive if the set of $f \in X^{*}$ which attain their norm on $S$ is norm dense in $X^{*}$; (clearly, $X$ is subreflexive if and only if $D(S)$ is norm dense in $S^{*}$ ).

E. Bishop and R. R. Phelps [1] have proved the significant result that every Banach space is subreflexive.

The following improves a result given by Šmulian [8, p. 550].

THEOREM 2. For a Banach space $X$, if the norm of $X^{*}$ is strongly differentiable on $S^{*}$ then $D(S)=S^{*}$ and $X$ is reflexive.

Proof. Since $X$ is a Banach space it is subreflexive. Therefore, for every $f \in S^{*}$ there exists a sequence $\left\{f_{n}\right\} \in D(S)$ which is norm convergent to $f$. Since the norm of $X^{*}$ is strongly differentiable there exists a unique support mapping $f \rightarrow F_{f}$ of $X^{*}$ into $X^{* *}$ and $\left\{\hat{x}_{n}\right\}$ is norm convergent to $F_{f}$ where $\hat{x}_{n}=F_{f_{n}} \in \hat{S}$ for $n=1,2, \ldots$ But $\hat{S}$ is complete, therefore $F_{f} \in \hat{S}$ and it follows that $f \in D(S)$ and $D(S)=S^{*}$. Since $X^{*}$ is smooth, the set of $F \in X^{* *}$ which attain their norm on $S^{*}$ is $\hat{X}$. Also since $X^{*}$ is a Banach space it is subreflexive. Therefore, $\hat{X}$ is norm dense in $X^{* *}$. But $\hat{X}$ is a norm closed linear subspace of $X^{* *}$. Therefore $\hat{X}=X^{* *}$, i.e. $X$ is reflexive.

The duality of uniform strong differentiability of the norm and uniform rotundity for a Banach space has been proved by Šmulian [7, p. 647]. By the 'duality' of these concepts we mean that the following theorem holds.

THEOREM 3. For a Banach space $X$,

(i) the norm of $X$ is uniformly strongly differentiable if and only if $X^{*}$ is uniformly rotund, and 
(ii) $X$ is uniformly rotund if and only if the norm of $X^{*}$ is uniformly strongly differentiable.

In Lemma 5 the equivalence of conditions (ii) and (iv) establishes (i) of Theorem 3. It follows from (i) of Theorem 3 that if the norm of $X^{*}$ is uniformly strongly differentiable then $X^{* *}$ is uniformly rotund and this implies that $X$ is uniformly rotund. The remainder of the proof will be given in Lemma 7, but to develop this we will need the following preliminary result.

LemMa 6. Let a Banach space $X$ have a support mapping $f \rightarrow F_{f}$ of $X^{*}$ into $X^{* *}$ such that on $D(S), F_{f}=\hat{x}$ where $\hat{x} \in D(f) \cap \hat{S}$. If this support mapping $f \rightarrow F_{f}$ is uniformly continuous on $D(S)$ when $X^{*}$ and $X^{* *}$ have the norm topologies, then $D(S)=S^{*}$.

Proof. Since $X$ is a Banach space it is subreflexive. Therefore, for every $f \in S^{*}$ there exists a sequence $\left\{f_{n}\right\} \in D(S)$ which is norm convergent to $f$. Since the support mapping $f \rightarrow F_{f}$ is uniformly continuous on $D(S),\left\{\hat{x}_{n}\right\}$ is a Cauchy sequence in $\hat{S}$ where $\hat{x}_{n}=F_{f_{n}} \in \hat{S}$ for $n=1,2, \cdots$. But $\hat{S}$ is complete, therefore there exists an $\hat{x} \in \hat{S}$ such that $\left\{\hat{x}_{n}\right\}$ is norm convergent to $\hat{x}$. Now

$$
\begin{aligned}
|f(x)-1| & \leqq\left|f(x)-f\left(x_{n}\right)\right|+\left|f\left(x_{n}\right)-f_{n}\left(x_{n}\right)\right| \\
& \leqq|| x-x_{n}\|+\| f-f_{n} \|,
\end{aligned}
$$

and so $f(x)=1$, i.e. $f \in D(S)$. Hence $D(S)=S^{*}$.

Following the same argument as that used in the proof of Lemma 5 , (iv) $\Rightarrow$ (i) above, we prove the associated result.

LEMMA 7. If a Banach space $X$ is uniformly rotund then the norm of $X^{*}$ is uniformly strongly differentiable.

Proof. Consider a support mapping $f \rightarrow F_{f}$ of $X^{*}$ into $X^{* *}$ such that on $D(S), F_{f}=\hat{x}$ where $\hat{x} \in D(f) \cap \hat{S}$. Using the primitive parallelogram law (3) for $X^{*}$, we have for $f_{1}, f_{2} \in D(S)$

$$
4 \leqq\left\|f_{1}+f_{2}\right\|\left\|\hat{x}_{1}+\hat{x}_{2}\right\|+\left\|f_{1}-f_{2}\right\|\left\|\hat{x}_{1}-\hat{x}_{2}\right\| \text {. }
$$

From the uniform rotundity of $X$ we derive that the support mapping $f \rightarrow F_{f}$ is uniformly continuous on $D(S)$. It follows from Lemma 6 and Theorem 1 (iii) that the norm of $X^{*}$ is uniformly strongly differentiable.

It should be noted that uniform strong differentiability of the norm and uniform rotundity are only truly dual in Banach spaces. Although it is evident that (i) of Theorem 3 holds for every normed linear space, the necessity condition for (ii) does not hold unless the space is complete. This can be seen from the fact that uniform rotundity of $X$ implies reflexivity only if $X$ is complete [9, p. 110], but from Lemma 5 the uniform strong differentiability of the norm of $X^{*}$ implies that $X^{* *}$ is uniformly rotund and consequently that $X$ is reflexive. 


\section{References}

[1] E. Bishop and R. R. Phelps, 'A proof that every Banach space is subreflexive', Bull. Amer. Math. Soc. 67 (1961), 97-98.

[2] F. F. Bonsall, B. E. Cain and H. Schneider, 'The numerical range of a continuous mapping of a normed space', Aeq. Math. 2 (1968), 86-93.

[3] D. F. Cudia, 'The geometry of Banach spaces. Smoothness', Trans. Amer. Math. Soc. 110 (1964), 284-314.

[4] H. Nakano, 'Topology and Linear Topological Spaces' (Maruzen, Tokyo, 1951).

[5] R. R. Phelps, 'A representation theorem for bounded convex sets', Proc. Amer. Math. Soc. $11(1960), 976-983$.

[6] V. L. Smulian, 'On some geometrical properties of the unit sphere in the space of type (B)', Mat. Sb. N.S. 48 (1938), 90-94.

[7] V. L. Smulian, 'Sur la dérivabilité de la norme dans l'espace de Banach', Dokl. Akad. Nauk. SSSR 27 (1940), 643-648.

[8] V. L. Smulian, 'Sur la structure de la sphère unitaire dans l'espace de Banach', Mat. Sb. N.S. 51 (1941), 545-561.

[9] A. Wilansky, 'Functional Analysis' (Blaisdell, New York, 1964).

The University of Newcastle, N.S.W. 